\title{
Discussion - Periodic variations and asteroseismology of active OB stars
}

\author{
Thomas Rivinius $^{1}$ and Juan Fabregat ${ }^{2}$ \\ ${ }^{1}$ ESO, Santiago, Chile \\ ${ }^{2}$ Observatorio Astronómico de la Universidad de Valencia, Calle Catedrático Agustín \\ Escardino 7, 46980 Paterna, Valencia, Spain
}

\begin{abstract}
We summarize the discussion held after the session on periodic variations and asteroseismology. The session not only included seven talks, but as well thirty excellent posters were shown. It was impossible to summarize all these in the available frame of a discussion, and so this work focuses on very few sub-topics only mentioned in the actual discussion session. These topics were the relation of pulsation and turbulence, pulsation in close binaries, the observed photometric variability, the connection of pulsations and outburst, and bipolar flows.
\end{abstract}

Keywords. stars: early-type, stars: emission-line, Be, stars: mass loss, stars: oscillations (including pulsations), line: profiles

\section{Pulsation and turbulence}

In this proceedings are several contributions on the relation between pulsation, in particular the pulsational pattern considering contributions from a set of periods varying in strength over time, and a line broadening parameter that was characterized as a type of turbulence (de Cat, Simón-Díaz et al., Godart et al., all this volume).

In order to set the discussion, first a reminder on the respective terms:

Microturbulence is a parameter, expressed as velocity, that mainly adds equivalent width to the intrinsic profile. The origin is more or less a still unknown, but possibly pulsation-related, velocity distribution related to the subsurface convection. Observationally, it finds its expression in e.g. the requirement to include such a parameter to obtain agreement on the temperature/gravity obtained from ionization balances of various elements, and of the same element over more than two ionization stages. As it is a local phenomenon, it does not change the global broadening function of the line profile.

Macroturbulence, in turn, are a sort of still unknown velocity fields over the total stellar surface. In the conventional treatment macroturbulence does not change the total equivalent width of a line, whereas micro turbulence of course does. The effect of macroturbulence is, however, to modify the shape of the line as observed in integrated light. This is most obvious in hot and luminous stars, for which already long ago a lack of low $v \sin i$ objects was found. Macroturbulence in this sense is an additional line broadening function.

In this context, it is important to remember that the line width parameter called $v \sin i$ is not directly the product of the equatorial rotational velocity with the sine of the inclination, but in fact a parameterization for the measured line-width, and various techniques to determine this parameters have to be carefully cross-calibrated in what they actually measure.

Despite the above description of local vs. global, an important technical point was raised 35 years ago by Gray (1975), who actually pointed out that the broadening 
mechanism for sharp line late type stars can be best described by what he called radial tangential microturbulence models. The physical basis was a picture in which the convection cells were envisioned as rectangular, and Gray described in that way how the broadening mechanism alters the line profiles: it put a lot of power in the wings and very little at small velocities, i.e. in the core. Indeed, if you take a look at the Fourier transform of the resulting line profile, it turns out that that gives you a very different signature from rotation compared to the one you would expect from microturbulence.

Now the interesting question is whether this also applies for hot type stars. Smith \& Gray (1976) actually worked on such a test, and came to the conclusion that indeed it seems to be that the same type of microturbulent description provides some basis to explain the observations. Nowadays, 35 years later not only there are much better observing techniques to probe (relatively) sharp lined hot stars much better, but the same is true for the analysis tools. It would be worthwhile to repeat the task done then, because this provides a powerful tool to see whether this type of microturbulence is important in relatively sharp lined hot stars.

Such studies have, in fact, been carried out on high resolution spectral time series of B supergiants to study the effect of microturbulence (Símon-Díaz and Herrero, 2007; Símon-Díaz et al., 2010). Applying the Fourier transform technique it was possible to disentangle rotation and the extra broadening. Very tight correlations exist between the size of the extra broadening, the line profile variations and the asymmetry of the lines, which support the claim that the additional broadening is caused by pulsations, oscillation or some similar kind of time variable phenomena.

For microturbulence, there is as of yet no physical explanation for the velocity field, nevertheless its order of magnitude has been measured with various techniques. When microturbulence is measured in the visual spectral domain, many people who use the OII lines to do so obtain supersonic values. On the other hand, this might be somewhat suspicious in particular for the more luminous objects. For instance, for luminosity class III stars, microturbulence of $20 \mathrm{~km} / \mathrm{s}$ is obtained sometimes, which is difficult to believe. On the other hand, it seems to be an often overlooked fact that, if one uses ultraviolet diagnostics, much lower microturbulence are usually inferred than those from the visual domain. In the past this has caused some debate until one was mutually convinced the respective other side just had a wrong measurement. However, after close inspection, this effect of the microturbulent velocity, scaling somehow with wavelength, is found to be a real one by Peters (this volume).

In the context of supersonic velocities, S. Owocki reminds that the first order assumption of the sonic velocity as an upper limit for pulsational or turbulent velocities might be misleading: Only when supersonic turbulence runs in small scale, it does quickly produce shocks and gets dissipated, as is supposed in the classical picture. However, on a large spatial scale velocities can well be supersonic and not produce shocks at all. For strictly ordered motions, like rotation, this is intuitively clear, but it is as well true for large scale turbulent (or pulsational) motions. What really counts is the divergence of the velocity field. Thus, if you have a large enough scale, there is no reason why even a turbulent velocity field cannot be supersonic.

\section{Pulsations in close binaries}

About 20 years ago, there was a thorough spectroscopic study on Spica ( $\alpha$ Vir) by Smith (1985a,1985b). Based on the moving bumps apparent in the spectral line profiles it was concluded that Spica shows non-radial pulsation, in a mode that consists of almost 
exclusive horizontal motions. In case of p-modes, the only way to understand this is in the frame of a Rossby wave, but for g-modes such motions are as well be possible for waves with long intrinsic periods (typically retrograde ones).

However, more recent studies, using modern techniques to confirm non-radial pulsations and mode identification, are lacking, and it seems in fact Spica has been ignored somewhat in terms of pulsation research, either because it was considered as "too bright" to be interesting, or because of its binary nature with a somewhat awkward 4day period, making it hard to get a complete phase diagram from a single observatory in less than a few years. Consequently, the most interesting question, namely whether and to what extent those bumps and the binarity are connected to each other is still unanswered.

\section{Photometric variability}

With all the new photometric data in hand, both from long term lensing projects as well as from the new satellite databases, an interesting exercise is to re-visit and compare these with what used to be the state-of-the art of our knowledge before we had such data-strings.

One question that is hoped to be answered with satellite data is that of the photometric variability of O-type stars. O stars display absorption components in the UV lines. Theoretically these can for instance be explained by the presence of bright spots in the surface of the star. If this is correct, however, O stars should present the photometric signature of rotational modulation, and this way the photometric data may give us the observational tools to advance on this question.

However, O stars are a too rare a species to have been caught by chance in number in the fields observed so far, and while one of the CoRoT fields focuses on a cluster with many $\mathrm{O}$ type stars, providing a good database for this kind of study, at the moment the results from this observation are still pending. Nevertheless, some first evidence from three stars point to rotational modulation an at least one of them, and a lot of incoherent variability, in the shape of red frequency noise, in the others. On the other hand, de Groot (this volume) does find evidence for solar-like oscillations in $\mathrm{O}$ type stars.

Another type of stars for which these data mean a breakthrough are the Be stars. In particular for those stars, 20 to 30 years ago, often claims of double- and even triplewave variability were made, i.e. variations that produce a better and less scattered phase diagram when they were sorted with twice or three times the period returned from a search for sinusoidal variations. As this terminology has somewhat disappeared from the discussion, one may wonder what might have happened to them, or are they all understood as multiperiodic variables nowadays? This is a particularly relevant question as such light curves were used as an argument to interpret short term variability as rotational modulation.

As it turned out, the non-observation of double wave periods with MOST is probably a selection effect, while they are clearly such light curves present in the CoRoT data. However, with the $150 \mathrm{~d}$ long time strings observed so far by CoRoT the answer to the above question is still difficult, as this is a relatively short time-base to resolve closely spaced periods. At least from the point of view of a formal analysis, one can say almost all stars indeed have a dominant frequency and the double of this frequency, i.e. its first harmonic. The amplitude of the first harmonic sometimes is higher, and sometimes weaker, than the first relevant frequency. 


\section{The pulsation-outburst connection}

Be stars have revealed interesting results as well in another respect: Some show a nice correlation between the excitation of the modes, or amplitude of the modes, and the beginning of an outburst. For some Be stars these outbursts have been confirmed spectroscopically, for some there is only photometric data, but the pattern is becoming increasingly clear: There is a connection between the spectroscopic outbursts, i.e. the ejection of material into the close circumstellar environment from the photosphere, and the short-period variability. Examples are HD 49330 (Huat et al., 2009), Corot 102719279 (Gutiérrez-Soto et al., 2010) and Achernar ( $\alpha$ Eri, Goss et al., 2010).

What is typically seen is that the overall photometric amplitude is high in times of outburst, and often additional modes become detectable as well. However, at this moment we only have a clear correlation, but the direction of the causality is unclear, i.e. we do not know whether it is the short periodic variability triggering, or even driving, the outburst, or whether it is rather the additional material in the close circumstellar environment altering the properties of the periodic variation, for instance through modification of the outer boundary condition.

Re-observing the stars for which such a correlation was found is crucial to answer the question of regularity for such outbursts and possibly to investigate the causality relation.

\section{Bipolar flows}

The conference logo itself, showing a star surrounded by a disk and having a bi-polar outflow, poses a question: Do these two properties co-exist in Be stars?

Observational data, obtained with interferometric techniques, supports such flows (e.g. on Achernar, Kervella \& Domiciano de Souza, 2006, or $\alpha$ Ara, Meilland et al., 2007). In more massive stars with a higher mass loss than main-sequence B stars not only winds are common but their concentration above the poles for rapid rotators are both theoretically understood and observationally confirmed: A wind gets concentrated towards the pole by very rapid rotation. However, these objects, O-type stars, do not have disks. Some B-type stars do, and while such a phenomenon as polar winds would certainly not be expected for normal B stars, this is less clear for Be stars.

The observations pointing to a polar wind were so far obtained with VINCI (which was a very reliable instrument) and AMBER (which at least at the time of the observations had its problems with absolute visibility calibration). Both instruments then worked in broad-band mode, however, in $\mathrm{K}$ or $\mathrm{H}+\mathrm{K}$, respectively, meaning they are sensitive only to changes of size in the continuum light. Unfortunately, other interferometric instruments could so far not confirm polar winds.

The optical thickness of such a polar wind to be seen in broad band interferometry, in any case, would have to be non-negligible. However, even if Be stars rotate at critical velocity, a theoretical, radiation driven wind based on a luminosity of the star would not be sufficiently dense, or in other words: the stellar poles even in a critically rotating star are not bright enough. Mass loss rates of at least equivalent to $10^{-5} \dot{\mathrm{M}}_{\odot} / \mathrm{yr}$, spread over a small area, would be necessary, and it is hardly feasible that a Be star can do anything close to that locally. Indeed, the known mass loss rates for main sequence B stars are much smaller, and main sequence normal mid-B stars, have almost no detectable wind at all.

In this context, it might be worthwhile to re-visit the IUE spectral database. For instance, in normal Be stars, the observed spectrum has quite weak SirII lines. Modern spectral synthesis codes reproduce the observed SiII lines quite nicely, so this line can 
be used as a diagnostic for the presence of polar winds in Be stars. One signature of the polar winds in Be stars should be emission in the ultraviolet SiII line. On the other hand, Grady, Bjorkman, \& Snow (1987) did not detect polar winds, but rather report enhanced winds above Be stars in intermediate latitudes.

\section{References}

Goss, K. J. F., Karoff, C., Chaplin, W. J., \& Elsworth, Y. et al. 2010, MNRAS tmp, 1668

Grady, C. A., Bjorkman, K. S., \& Snow, T. P. 1987, ApJ, 320, 376

Gray, D. F. 1975, ApJ, 202, 148

Gutiérrez-Soto J., Semaan T., Garrido R., \& Baudin F. et al. 2010, AN 331, P51, arXiv:1010.1910

Huat, A.-L., Hubert, A.-M., Baudin, F., \& Floquet, M. et al. 2009, A 6 A, 506, 95

Kervella, P. \& Domiciano de Souza, A. 2006, A\& A, 453, 1059

Meilland, A., Stee, P., Vannier, M., \& Millour, F. et al. 2007, A\&A A, 464, 59

Simón-Díaz, S., Herrero, A., Uytterhoeven, K., \& Castro, N. et al. 2010, ApJ (Letters), 720, L174

Simón-Díaz, S. \& Herrero, A. 2007, A\&A, 468, 1063

Smith, M. A. 1985a, ApJ, 297, 206

Smith, M. A. 1985b, ApJ, 297, 224

Smith, M. A. \& Gray, D. F. 1976, PASP, 88, 809 